\title{
Effect of jasmonic acid on glucosinolate metabolism in different organs of broccoli sprouts
}

\author{
Yinglian Zhu, Fengwu Wang, Liping Guo* \\ College of Food Science and Engineering, Qingdao Agricultural University, Qingdao, 266109, P.R. China
}

\section{A B S T R A C T}

\begin{abstract}
Edible sprouts, especially Brassica sprouts, contain high levels of health-promoting compounds. Exogenous elicitors have been used as strategies to improve the nutraceutical quality of Brassica sprouts. In this study, effects of jasmonic acid (JA) treatment on growth, the levels of glucosinolates and isothiocyanates, as well as myrosinase activity in different organs of broccoli sprouts were investigated. JA treatment markedly increased the contents of glucosinolates (GSLs), especially glucoraphanin, glucobrassicin and neoglucobrassicin in broccoli sprouts. However, gluconapin was not affected even decreased by JA treatment. Cotyledon, hypocotyl and root obtained the different results in induction of GSLs. Among these, neoglucobrassicin obtained the highest enhancement in three organs. Myrosinase activity in cotyledon of broccoli increased after JA treatment, while decreased in hypocotyl. Three concentrations of JA all significantly increased sulforaphane and isothiocyanates formation in cotyledon, hypocotyl and root of broccoli sprouts. Application of $100 \mu \mathrm{M}$ JA led to the highest myrosinase activity, the least gluconapin and the most sulforaphane and isothiocyanates in cotyledon, as well as the most isothiocyanates in root. These results indicated that JA treatment could be an effective way to improve the cancer-prevention benefits of broccoli sprouts via enhancing sulforaphane and total isothiocyanates.
\end{abstract}

Keywords: Broccoli sprouts; Jasmonic acid; Glucosinolates; Isothiocyanates; Myrosinase

\section{INTRODUCTION}

Brassica vegetables are recognized as wellness and health-promoting foods because of their high levels of bioactive compounds, such as vitamin C, carotenoids, tocopherols, polyphenolics and glucosinolates $\left(\mathrm{GSL}_{\mathrm{s}}\right)$

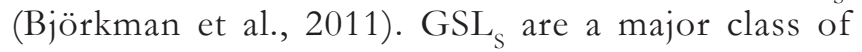
nitrogen- and sulfur-containing secondary metabolites involved in Brassica vegetable defense against pathogens (Bell and Wagstaff, 2017; Gu et al., 2012). When the plant tissue is disrupted, glucosinolates could be hydrolyzed into isothiocyanates (ITCs), thiocyanates, nitriles, epithionitriles and oxazolidines by the action of myrosinase (Bones and Rossiter, 2006; Gu et al., 2012). Among these compounds, ITCs, especially sulforaphane, displays diverse and important physiological activities including carcinogen detoxification, reducing the risk of cardiovascular diseases, anti-inflammatory and inhibition of pathogenic fungal growth as well as reducing blood glucose, etc. (Axelsson et al., 2017; Fahey et al., 2017; Guo et al., 2018).
Broccoli (Brassica oleracea var. italica) is widely consumed Brassica vegetable not just in China but all over the world. Moreover, broccoli sprouts contain much higher level of GSL $_{\mathrm{s}}$ than adult vegetables (Fahey et al., 1997). Hence, consumption of broccoli sprouts has become an popular way for people to enhance and keep their health status (Nguyen et al., 2016; Nguyen et al., 2017). Plant hormones have been used as elicitors to increase accumulation of bioactive compounds in plants (Guo et al., 2014c; Kim et al., 2006; Pérez-Balibrea et al., 2011). Jasmonic acid (JA) is generally acted as a growth regulator derived largely from linolenic acid through an octadecanoid pathway. In addition, exogenous JA also can activate plant defense gene either by acting directly on the genes or the octadecanoid pathway (Koo and Howe, 2009). JA has been used as a powerful inducer to enhance glucosinolate content in many plants (Baenas et al., 2014; Kastell et al., 2013). Addition of exogenous JA enhanced indole $\mathrm{GSL}_{\mathrm{S}}$ content, particularly 1-methoxyindolyl-3-methyl glucosinolate in hairy root cultures of Sinapis alba and Brassica rapa (Kastell et al., 2013). Additionally, application of $150 \mu \mathrm{M}$ JA also

\footnotetext{
${ }^{*}$ Corresponding author:

Liping Guo, College of Food Science and Engineering, Qingdao Agricultural University, Qingdao, 266109, P.R. China.
}

Tel: + 86-0532-86080771. E-mail: happyglp@126.com

Received: 02 December 2018; $\quad$ Accepted: 31 January 2019 
increased glucosinolate content in Brassicaceae sprouts, for instance glucobrassicin in turnip and rutabaga sprouts, glucoraphenin in radish sprouts and glucoraphanin in broccoli sprouts (Baenas et al., 2014). However, the effects of JA treatment on sulforaphane and ITCs formation as well as myrosinase activity in broccoli sprouts were not investigated in aforementioned studies.

Therefore, the objective of the present work was to investigate the changes in glucosinolate content, myrosinase activity and the production of ITCs in cotyledon, hypocotyl and root of broccoli sprouts after treatment with JA.

\section{MATERIAL AND METHODS}

\section{Plant material, seed germination and treatment}

Broccoli seeds were supplied by Shouguang Wentian Seed Co. (Shandong, China). After sterilizing with $5 \mathrm{~mL} / \mathrm{L}$ sodium hypochlorite, the seeds were placed in distilled water and soaked for $4 \mathrm{~h}$ at $30^{\circ} \mathrm{C}$. Then, they were weighed and spread evenly on trays filled with vermiculite as a substrate irrigated with distilled water. All sprouts with three replicates were grown in a growth chamber with a $16 \mathrm{~h}$ light $/ 8 \mathrm{~h}$ dark cycle at $25^{\circ} \mathrm{C}$. JA was dissolved in $0.2 \%$ ethanol and sprayed on sprouts at $100 \mu \mathrm{M}, 200 \mu \mathrm{M}$, $300 \mu \mathrm{M}$ concentrations every $24 \mathrm{~h}$ after one day. The control sprouts were sprayed with $10 \mathrm{~mL}$ distilled water. Finally, the cotyledon, hypocotyl and root were rapidly and gently dissected from 5 -days old sprouts and then frozen at $-80^{\circ} \mathrm{C}$ for measurements.

\section{Extraction and determination of $\mathrm{GSL}_{\mathrm{s}}$}

A modified version of a previously reported procedure by Font et al. (2005) and Guo et al. (2014a) was used for the extraction and analysis of $\mathrm{GSL}_{\mathrm{s}}$. GSL $\mathrm{s}$ were extracted twice with boiling $75 \%$ methanol. After purification with DEAE-Sephadex A-25 column (acetic acid activated), desulpho-GSL ${ }_{\mathrm{S}}$ were obtained by the addition of aryl sulfatase (Sigma-Aldrich, St. Louis, MO, USA) solution and incubation at $35^{\circ} \mathrm{C}$ overnight. An Agilent $1200 \mathrm{HPLC}$ system (Agilent Technologies Co. Ltd., USA) equipped with an Eclipse XDB-C18 column (5 $\mu \mathrm{m}$ particle size, 4.6 $\times 150 \mathrm{~mm}$ ) was used to analyze $\mathrm{GSL}_{\mathrm{S}}$.

\section{Myrosinase activity assay}

The method of Guo et al. (2014b) was applied to assay myrosinase activity. The myrosinase activity corresponded to the amount of glucose formed per minute and milligram of protein. The previous procedure of Bradford (1976) was used to determine the protein content of the supernatant.

\section{Sulforaphane determination}

A previously described method for sulforaphane analysis was used in this study (Guo et al., 2014a). Broccoli sprouts
(500 mg) were hydrolyzed at $37^{\circ} \mathrm{C}$ for $3 \mathrm{~h}$ by endogenous myrosinase, then were extracted with dichloromethane. After concentrating on a rotary evaporator, the residue was dissolved in 10\% acetonitrile and filtered through a $0.45 \mathrm{~mm}$ membrane before HPLC analysis. The sulforaphane production was expressed as $\mathrm{mg} / 100 \mathrm{~g}$ fresh weight of broccoli sprouts.

\section{Total ITCs determination}

The extraction method of ITCs was in accordance with that of sulforaphane extraction. The dichloromethane fraction containing ITCs was concentrated to about $1 \mathrm{~mL}$. The determination of total ITCs was carried out following the previous procedure described by Tang et al. (2013) and Wang et al. (2015). The reaction mixture consisted of $1 \mathrm{~mL}$ of the dichloromethane fraction, $2 \mathrm{~mL}$ of methanol, $0.2 \mathrm{~mL}$ of $7 \mathrm{mmol} / \mathrm{L} \mathrm{1,2-benzenedithiol} \mathrm{and} 1.8 \mathrm{~mL}$ of $50 \mathrm{mmol} / \mathrm{L}$ sodium borate buffer ( $\mathrm{pH} 8.5$ ). After reacting for $1 \mathrm{~h}$ at $65^{\circ} \mathrm{C}$, the mixture was centrifuged for $10 \mathrm{~min}$ at $5000 \mathrm{~g}$. Analyses of ITCs were conducted on an Agilent 1200 HPLC system (Agilent Technologies Co. Ltd., USA) equipped with an Eclipse XDB-C18 column and a G1314B UV detector at $365 \mathrm{~nm}$. Assay was performed at a flowrate of $1.75 \mathrm{~mL} / \mathrm{min}$ with $70 \%$ methanol and $30 \% \mathrm{H}_{2} \mathrm{O}$. Production of ITCs was expressed as $\mathrm{mg} / 100 \mathrm{~g}$ fresh weight of broccoli sprouts.

\section{Statistical analyses}

All statistical analyses were performed with SPSS 18.0 (SPSS Inc., Chicago, IL, USA) in our experiment. Data was analyzed by two-way ANOVA with treatment and organ as factors, followed by Duncan's multiple comparison test. Differences at $p<0.05$ were considered as statistically significant.

\section{RESULTS}

\section{Effect of JA on sprout length and fresh weight of broccoli sprouts}

The length and fresh weight of broccoli sprouts were both significantly decreased after all JA treatments $(p<0.05)$ (Fig. $1 \mathrm{~A}$ and B). The highest decrease in length and fresh weight of broccoli sprouts was observed under $300 \mu \mathrm{M}$ JA treatment, in comparison with the control sample. No significant difference was found in fresh weight between $100 \mu \mathrm{M}$ and $200 \mu \mathrm{M}$ JA treatment.

\section{Effect of $\mathrm{JA}$ on $\mathrm{GSL}_{\mathrm{s}}$ contents in cotyledon, hypocotyl and root of broccoli sprouts}

Seven kinds of $\mathrm{GSL}_{\mathrm{S}}$, including three aliphatic $\mathrm{GSL}_{\mathrm{S}}$ and four indole $\mathrm{GSL}_{\mathrm{S}}$ were detected in difference organs of broccoli sprouts (Fig. 2 and Fig. 3). The influence of JA treatment on individual GSL profile differed considerably. Application of JA significantly enhanced glucoraphanin 


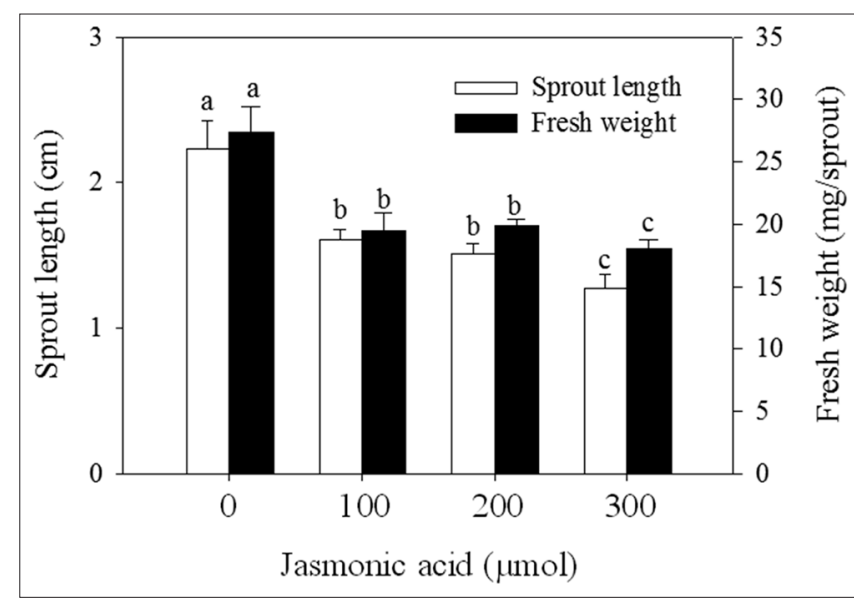

Fig 1. Sprout length, fresh weight of broccoli sprouts under JA treatment. Each point was expressed as mean $\pm S D(n=3)$. Values not sharing the same letter at the same index are significantly different at $p<0.05$.

and glucoerucin content but did not affect even reduced gluconapin content in cotyledon (Fig. 2). Additionally, no significant difference was found in aliphatic GSL content in cotyledon among the three concentrations of JA.

On the other hand, JA treatment was found more effective for increasing indole $\mathrm{GSL}_{\mathrm{S}}$ than aliphatic ones, especially glucobrassicin and neoglucobrassicin. The level of neoglucobrassicin in cotyledon, hypocotyl and root treated with $200 \mu \mathrm{M}$ JA was $53.44,49.67$ and 13.12 times of that in the control samples, respectively. However, only glucobrassicin content in cotyledon was dramatically enhanced by JA treatment, which increased up to 7.67- to 8.91-fold of that in control cotyledon. Except neoglucobrassicin, other indole $\mathrm{GSL}_{\mathrm{S}}$ in root was not affected by JA treatment.

Effect of JA on myrosinase activity in cotyledon, hypocotyl and root of broccoli sprouts

Compared to the control, JA treatment increased myrosinase activity in cotyledon but decreased it in hypocotyl and root. On the other hand, the myrosinase activity in hypocotyl and root continuously decreased with the increase of JA concentration. After treatment with $100 \mu \mathrm{M} \mathrm{JA}$, compared to the control, myrosinase activity in cotyledon increased by $95.4 \%$, but in hypocotyl and root decreased by $12.1 \%$ and $20.7 \%$, respectively (Fig. 4).

Effect of JA on sulforaphane and ITCs formation in cotyledon, hypocotyl and root of broccoli sprouts

JA treatments dramatically increased the formation of sulforaphane and ITCs in broccoli sprouts $(p<0.05)$. While in cotyledon, there was no significant difference in sulforaphane formation among three concentrations (Fig. $5 \mathrm{~A}$ ); however, $100 \mu \mathrm{M} \mathrm{JA}$ showed more ITCs formation than $200 \mu \mathrm{M}$ and $300 \mu \mathrm{M}$ JA (Fig. 5 B). In addition, no significant difference
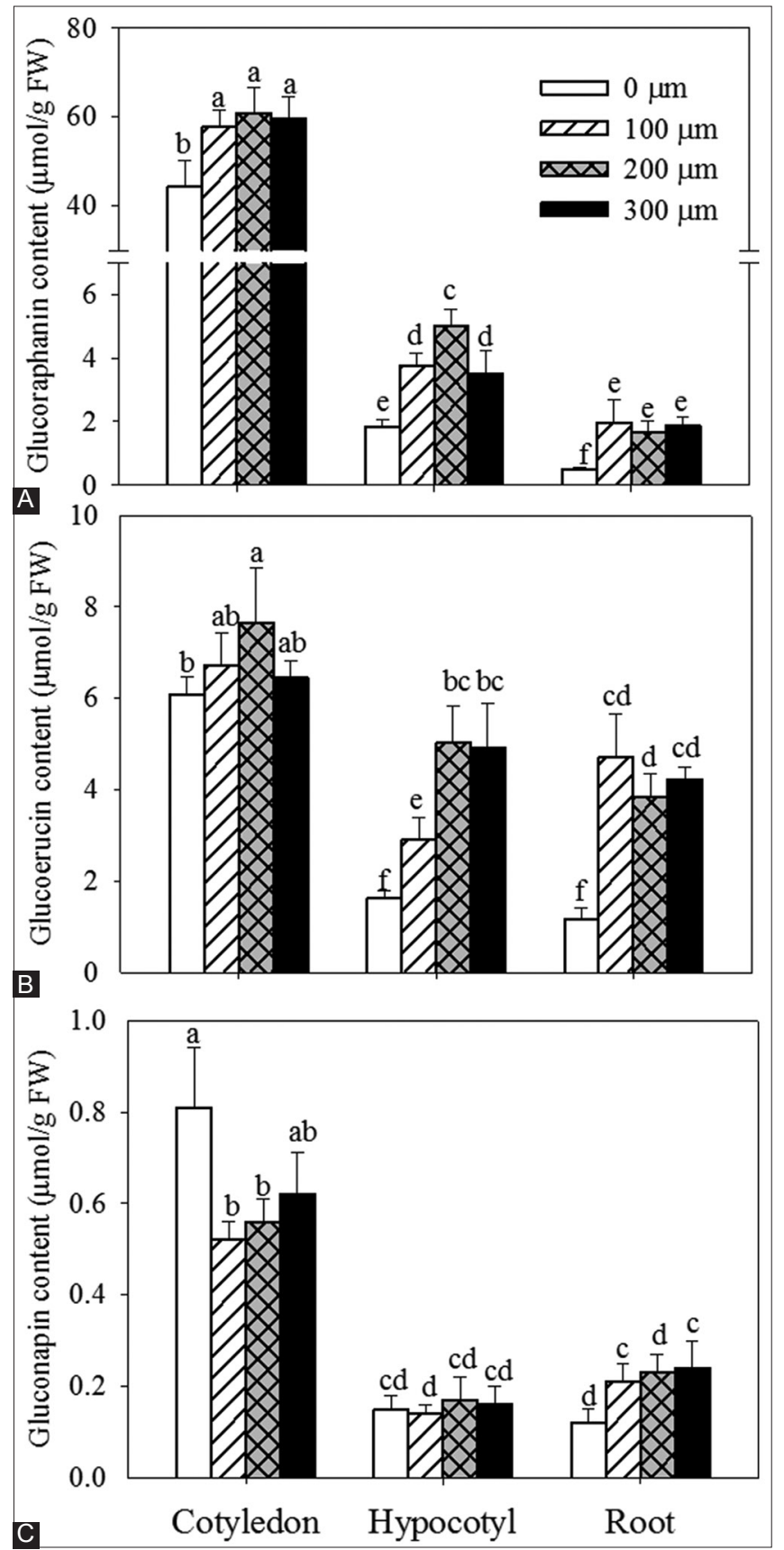

Fig 2. (A-C) Aliphatic glucosinolate content in different organs of broccoli sprouts under JA treatment. Each point was expressed as mean $\pm S D(n=3)$. Values not sharing the same letter are significantly different at $p<0.05$.

was obtained in sulforaphane and ITCs formation in hypocotyl between $200 \mu \mathrm{M}$ and $300 \mu \mathrm{M} \mathrm{JA}$. Root contained the lowest sulforaphane and ITCs.

\section{DISCUSSION}

In the present study, the growth of broccoli sprouts measured as sprout length and fresh weight, which was markedly decreased by JA treatment when compared to 


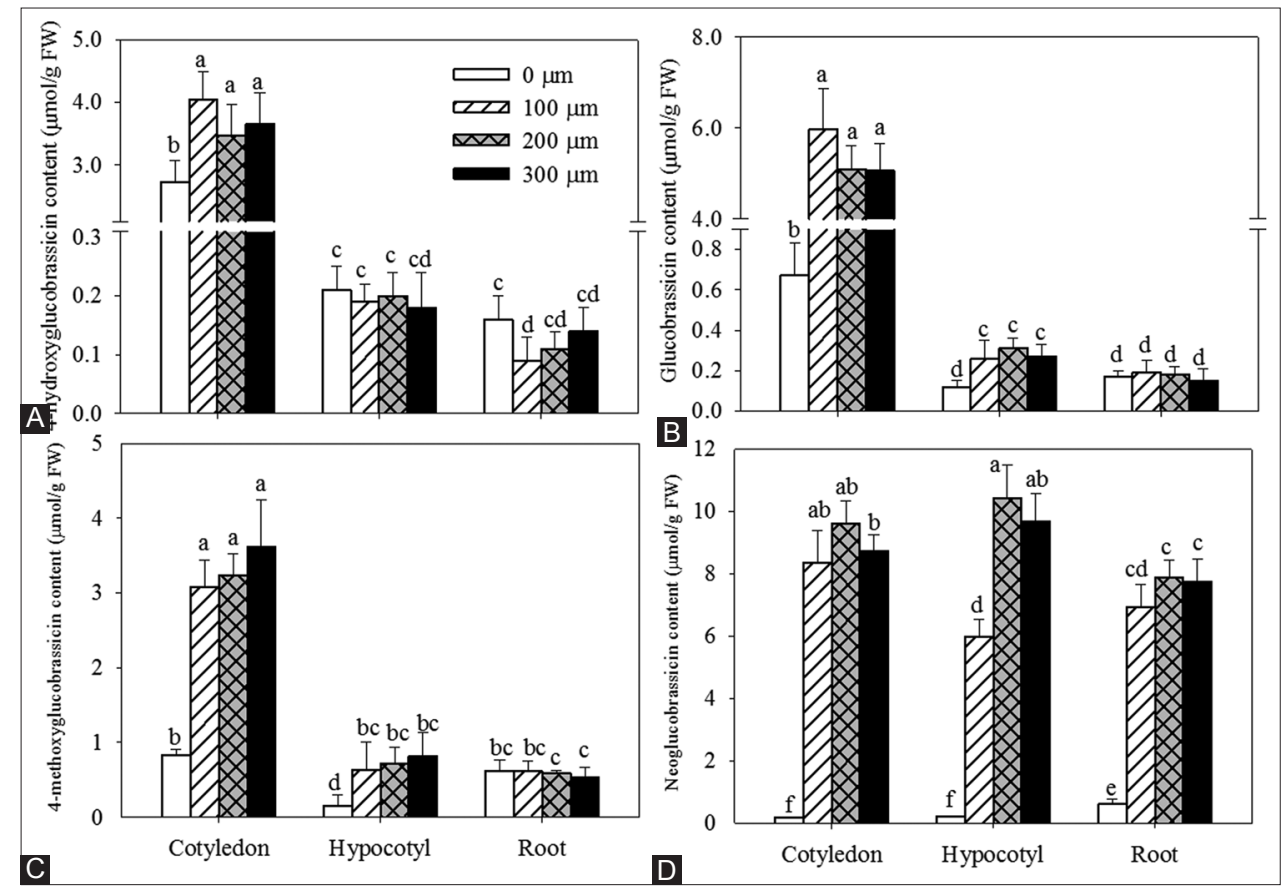

Fig 3. (A-D) Indole glucosinolate content in different organs of broccoli sprouts under JA treatment. Each point was expressed as mean \pm SD $(\mathrm{n}=3)$. Values not sharing the same letter are significantly different at $p<0.05$.

the control. This result was in line with the findings by Kastell et al. (2013) and Baenas et al. (2014), which showed that addition of JA significantly decreased the fresh weight and biomass in hairy root and Brassicaceae sprouts such as broccoli, rutabaga, turnip and radish.

Broccoli sprouts are rich in glucoraphanin, which can be hydrolyzed into the corresponding ITC, sulforaphane by the action of myrosinase (Guo et al., 2018). In our study, the most substantial glucosinolate in broccoli sprouts was glucoraphanin, accounting for $72 \%$ of total glucosinolate content, which was in consistent with the results of Guo et al. (2011) and Pérez-Balibrea et al. (2011). However, it was different from the findings of Guo et al. (2014c) and Sun et al. (2015), who found that the glucoerucin, not glucoraphanin was the most abundant one. Glucoerucin is the precursor of glucoraphanin and can be converted to glucoraphanin by the hydrolysis of the enzyme encoding of GSL-OX (Li et al., 2008). Hence, the different phenomenon might be attribute to the different activities of the enzyme GSL-OX (Guo et al., 2014c). Additionally, previous studies found that glucosinolate composition and concentration varied considerably among different organs within the same plant and a plant species (Brown et al., 2003; Pérez-Balibrea et al., 2008). The results of this study indicated that the cotyledon of broccoli sprouts contained the highest glucosinolate content except neoglucobrassicin.

Many factors can dramatically induced biosynthesis of glucosinolates, such as wounding, phytohormone,

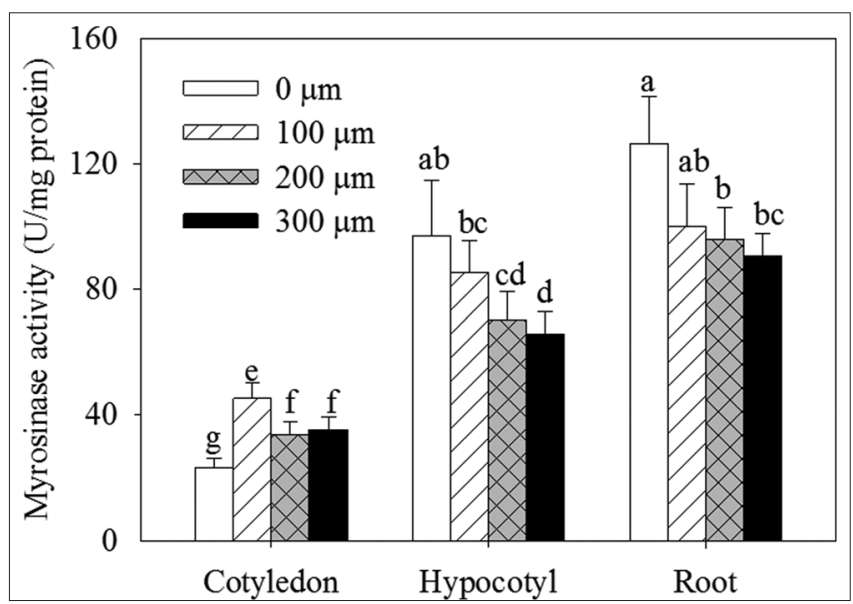

Fig 4. Myrosinase activity in different organs of broccoli sprouts under $J A$ treatment. Each point was expressed as mean $\pm S D(n=3)$. Values not sharing the same letter are significantly different at $p<0.05$.

pathogen, herbivore and sugar, etc. (Gu et al., 2012). JA as an elicitor and signal molecule has been used successfully in different plants to improve production of $\mathrm{GSL}_{\mathrm{S}}$ (Baenas et al., 2014; Kastell et al., 2013). In the present study, application of JA increased the concentration of GSL, such as glucoraphanin, glucoerucin, glucobrassicin and neoglucobrassicin in three organs. Particularly, neoglucobrassicin obtained the maximum increase in sprouts, which was in accordance with the finding in Hairy Root of Brassica rapa by Kastell et al. (2013), who found that neoglucobrassicin content enhanced by about 16 times on day 14 after application of $100 \mu \mathrm{M} \mathrm{JA}$. Interestingly, JA had no effect or even decreased the concentration 


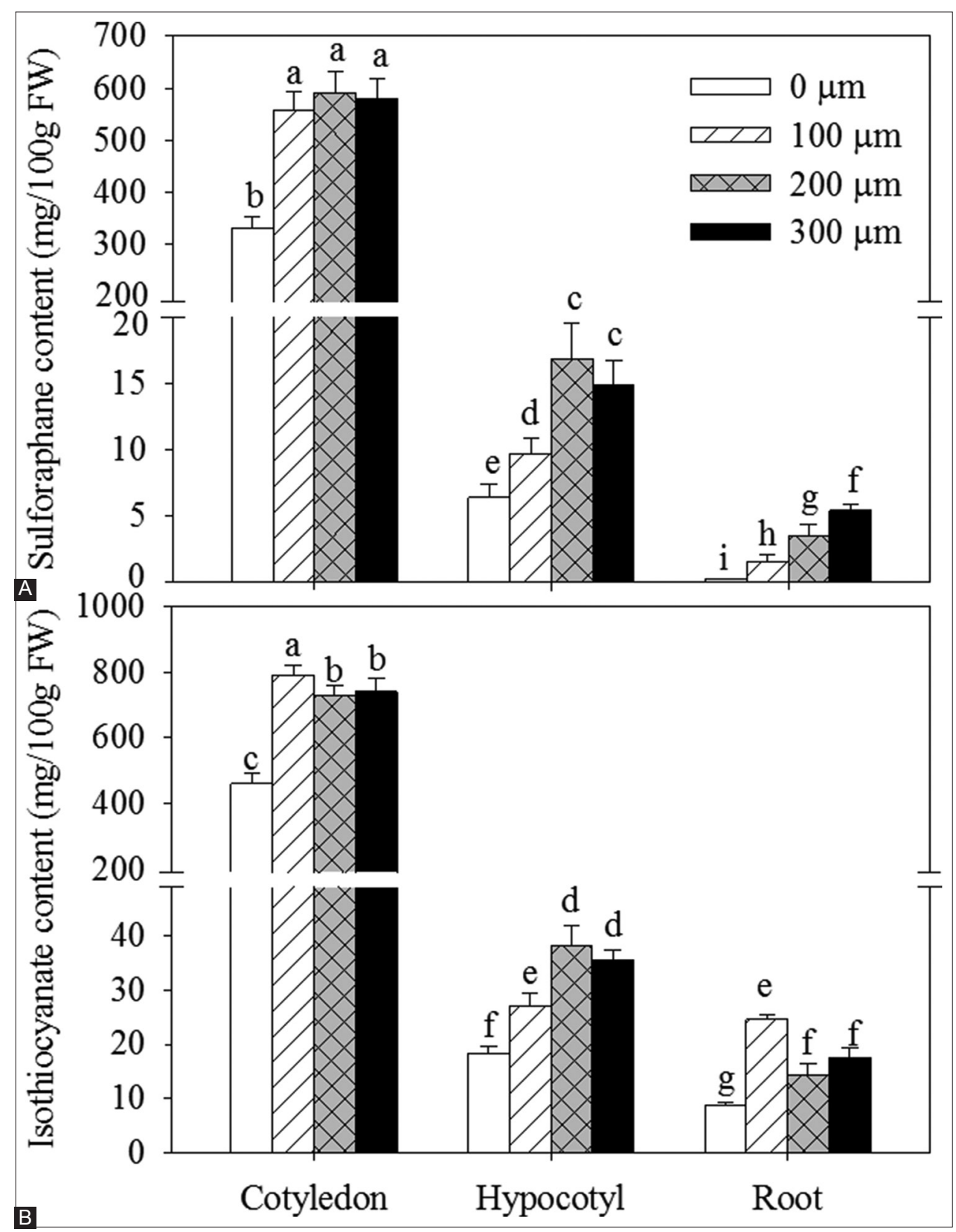

Fig 5. The formation sulforaphane (A) and ITCs (B) in different organs of broccoli sprouts under JA treatment. Each point was expressed as mean $\pm S D(n=3)$. Values not sharing the same letter are significantly different at $p<0.05$.

of gluconapin. A substantial increase of glucobrassicin content was only found in cotyledon of broccoli sprouts after JA treatments. Additional researches are needed to expound the underlying mechanism of different effects of JA on different $\mathrm{GSL}_{\mathrm{S}}$ and organs.

Different effects of JA treatment on myrosinase activity in different organs were also detected in the present study. Myrosinase activity in hypocotyl and root reduced after JA treatments. The result was similar to the result of Kim et al. (2006), who reported that jasmonates hormone (methyl jasmonate) decreased myrosinase activity in radish sprouts. However, myrosinase activity in cotyledon significantly enhanced after JA treatments. ITCs, hydrolyzed from aliphatic and aromatic GSLs, are thought to be accountable for decreasing the risk of in the development of various types of cancer (Traka and Mithen, 2009; Zhang, 2004). In addition, the ITCs formed from indole GSLs are unstable, but the hydrolysis products of indole GSLs, in particular indole-3-carbinol, have the potential anticancer activity (Fahey et al., 2001). Sulforaphane, an isothiocyanate hydrolyzed from glucoraphanin, has received the most attention. In addition, the enzymolytic ITCs from gluconapin and glucoerucin are 1-butene-4-isothiocyanat and erucin, which also presented anticancer effects in vitro and in vivo (Melchini and Traka, 2010; Morroni et al., 2018). 
In the present study, sulforaphane concentration accounted for $62.59 \%$ of total ITCs in broccoli sprouts. As expected, three concentrations of JA all enhanced the formation of sulforaphane and ITCs in cotyledon, hypocotyl and root of broccoli sprouts, which was in accordance with changing trend of the content of precursor GSLs. Besides, there was no significant difference in the level of total aliphatic GSLs in cotyledon of broccoli sprouts among the three concentrations of JA, but $100 \mu \mathrm{M} \mathrm{JA}$ treatment led to more ITCs formation in cotyledon than $200 \mu \mathrm{M}$ and $300 \mu \mathrm{M}$ JA treatment. The probable explanation for this phenomenon was the fact that myrosinase activity in cotyledon treated with $100 \mu \mathrm{M} \mathrm{JA}$ was higher than that of $200 \mu \mathrm{M}$ and $300 \mu \mathrm{M} \mathrm{JA}$ treatment. Further research will be carried out to investigate the induction of hydrolysis products of indole GSLs by JA treatment and the molecular mechanisms involved.

\section{CONCLUSIONS}

In conclusion, except gluconapin, the content of other GSLs in broccoli sprouts increased after JA treatment. However, the different effects were found in different organs and different GSLs as well as JA concentrations. No significant difference was found in all individual GSL content in cotyledon among $100 \mu \mathrm{M}, 200 \mu \mathrm{M}$ and $300 \mu \mathrm{M}$ JA. In particular, JA treatment resulted in the highest enhancement of neoglucobrassicin content in cotyledon, hypocotyl and root. Treatment with JA enhanced myrosinase activity in cotyledon but reduced it in hypocotyl and root. Besides, the yield of ITCs and sulforaphane in three organs also increased after JA treatment, which was similar to changing trend of the content of glucoraphanin and aliphatic GSLs. Cotyledon contained the majority of GSLs and ITCs both in control sample and in JA treated sprouts. In summary, treatment with JA is an effective strategy for selective increase the bioactive compounds GSLs and ITCs in broccoli sprouts and is potential for the production and commercialization of functional sprouts.

\section{ACKNOWLEDGEMENTS}

This work was supported by the financial support provided by the National Natural Science Foundation of China (No. 31801457), the Natural Science Foundation of Shandong Province of China (No. ZR2016CQ17) and the Advanced Talents Foundation of Qingdao Agricultural University (No. 6631115051).

\section{Authors' contributions}

Y.Z. and L.G. conceived designed the study; Y.Z., F.W. and L.G. carried out the experiments; F.W. analyzed the data; Y.Z. and L.G. wrote the manuscript. All authors read and approved the final manuscript.

\section{REFERENCES}

Axelsson, A. S., E. Tubbs, B. Mecham, S. Chacko, A. N. Hannah, Y. Tang, J.W. Fahey, J. M. J. Derry, C. B. Wollheim, N. Wierup, M. W. Haymond, S. H. Friend, H. Mulder and A. H. Rosengren. 2017. Sulforaphane reduces hepatic glucose production and improves glucose control in patients with Type 2 diabetes. Sci. Transl. Med. 9: eaah4477.

Baenas, N., C. Garcia-Viguera and D. A. Moreno. 2014. Biotic elicitors effectively increase the glucosinolates content in Brassicaceae sprouts. J. Agric. Food Chem. 62: 1881-1889.

Bell, L., and C. Wagstaff. 2017. Enhancement of glucosinolate and isothiocyanate profiles in Brassicaceae crops: Addressing challenges in breeding for cultivation, storage, and consumer related traits. J. Agric. Food Chem. 65: 9379-9403.

Björkman, M., I., Klingen, A. N. E. Birch, A. M. Bones, T. J. A. Bruce, T. J. Johansen, R. Meadow, J. Mølmann, R. Seljåsen, L. E. Smart and D. Stewart. 2011. Phytochemicals of Brassicaceae in plant protection and human health-influences of climate, environment and agronomic practice. Phytochemistry. 72: 538-556.

Bones, A. and J. Rossiter. 2006. The enzymic and chemically induced decomposition of glucosinolates. Phytochemistry. 67: 1053-1067.

Bradford, M. M. 1976. A rapid and sensitive method for thequantitation of microgram quantities of protein utilizing the principle of protein-dye binding. Anal. Biochem. 72: 248-254.

Brown, P. D., J. G. Tokuhisa, M. Reichelt and J. Gershenzon. 2003. Variation of glucosinolate accumulation among different organs and developmental stages of Arabidopsis thaliana. Phytochemistry. 62: 471-481.

Fahey, J. W., K. L. Wade, S. L. Wehage, W. D. Holtzclaw, H. Liu, P. Talalay, E. Fuchs and K. K. Stephenson. 2017. Stabilized sulforaphane for clinical use: Phytochemical delivery efficiency. Mol. Nutr. Food Res. 61: 1600766.

Fahey, J. W., A. T. Zalcmann and P. Talalay. 2001. The chemical diversity and distribution of glucosinolates and isothiocyanates among plants. Phytochemistry. 56: 5-51.

Fahey, J. W., Y. Zhang and P. Talalay. 1997. Broccoli sprouts: An exceptionally rich source of inducers of enzymes that protect against chemical carcinogens. Proc. Natl. Acad. Sci. 94: 10367-10372.

Font, R., M. Río-Celestino, E. Cartea and A. de Haro-Bailón. 2005. Quantification of glucosinolates in leaves of leaf rape (Brassica napus ssp. Pabularia) by near-infrared spectroscopy. Phytochemistry. 66: 175-185.

Gu, Z., Q. Guo and Y. Gu. 2012. Factors influencing glucoraphanin and sulforaphane formation in Brassica plants: A review. J. Integr. Agric. 11: 1804-1816.

Guo, L., R. Yang, Z. Wang, Q. Guo and Z. Gu. 2014a. Glucoraphanin, sulforaphane and myrosinase activity in germinating broccoli sprouts as affected by growth temperature and plant organs. J. Funct. Foods. 9: 70-77.

Guo, L., R. Yang, Z. Wang, Q. Guo and Z. Gu. 2014b. Effect of $\mathrm{NaCl}$ stress on health-promoting compounds and antioxidant activity in the sprouts of three broccoli cultivars. Int. J. Food Sci. Nutr. 65: 476-481.

Guo, L., Y. Zhu and F. Wang. 2018. Calcium sulfate treatment enhances bioactive compounds and antioxidant capacity in broccoli sprouts during growth and storage. Postharvest Biol. Technol. 139: 12-19.

Guo, R., Q. Hou, G. Yuan, Y. Zhao and Q. Wang. 2014c. Effect of 2, 4-epibrassinolide on main health-promoting compounds in 
broccoli sprouts. LWT Food Sci. Technol. 58: 287-292.

Guo, R., G. Yuan and Q. Wang. 2011. Effect of sucrose and mannitol on the accumulation of health-promoting compounds and the activity of metabolic enzymes in broccoli sprouts. Sci. Hortic. 128: $159-165$.

Kastell, A., I. Smetanska, C. Ulrichs, Z. Cai and I. Mewis. 2013. Effects of phytohormones and jasmonic acid on glucosinolate content in hairy root cultures of Sinapis alba and Brassica rapa. Appl. Biochem. Biotechnol. 169: 624-635.

Kim, H. J., F. Chen, X. Wang and J. H. Choi. 2006. Effect of methyl jasmonate on phenolics, isothiocyanate, and metabolic enzymes in radish sprout (Raphanus sativus L.). J. Agric. Food Chem. 54: 7263-7269.

Koo, A. J. K. and G. A. Howe. 2009. The wound hormone jasmonate. Phytochemistry. 70: 1571-1580.

Li, J., B. G. Hansen, J. A. Ober, D. J. Kliebenstein and B. A. Halkier. 2008. Subclade of flavin-monooxygenases involved in aliphatic glucosinolate biosynthesis. Plant Physiol. 148: 1721-1733.

Melchini, A. and M. Traka. 2010. Biological profile of rrucin: A new promising anticancer agent from cuciferous vegetables. Toxins. 2: 593-612.

Morroni, F., G. Sita, A. Djemil, M. D’Amico, L. Pruccoli, G. CantelliForti, P. Hrelia and A. Tarozzi. 2018. Comparison of adaptive neuroprotective nechanisms of sulforaphane and its interconversion poduct eucin in in vitro and in vivo models of Parkinson's disease. J. Agric. Food Chem. 66: 856-865.

Nguyen, A. T., A. M. A. Bahry, K. Q. Shen, E. A. Armstrong and J. Y. Yager. 2016. Consumption of broccoli sprouts during late gestation and lactation confers protection against developmental delay induced by maternal inflammation. Behav. Brain Res. 307: 239-249.

Nguyen, B. A. V., G. McDonald, F. Fiorentino, B. C. Reeves, J. Kwak, S. Pyo, G. D. Angelini, J. R. Anderson, G. Frost, D. O. Haskard and P. C. Evans. 2017. Consumption of broccoli sprouts attenuates intracellular P38 map kinase and reactive oxygen species pro-inflammatory activation in human leukocytes: A randomised-controlled trial. J. Clin. Nutr. Diet. 3: 25.

Pérez-Balibrea, S., D. A. Moreno and C. García-Viguera. 2011. Improving the phytochemical composition of broccoli sprouts by elicitation. Food Chem. 129: 35-44.

Sun, J., L. Kou, P. Geng, H. Huang, T. Yang, Y. Luo and P. Chen. 2015. Metabolomic assessment reveals an elevated level of glucosinolate content in $\mathrm{CaCl}_{2}$ treated broccoli microgreens. J. Agric. Food Chem. 63: 1863-1868.

Tang, L., J. D. Paonessa, Y. Zhang, C. B. Ambrosone and S. E. McCann. 2013. Total isothiocyanate yield from raw cruciferous vegetables commonly consumed in the United States. J. Funct. Foods. 5: 1996-2001.

Traka, M. and R. Mithen. 2009. Glucosinolates, isothiocyanates and human health. Phytochem. Rev. 8: 269-282.

Wang, Z., R. Yang, L. Guo, M. Fang, Y. Zhou and Z. Gu. 2015. Effects of abscisic acid on glucosinolate content, isothiocyanate formation and myrosinase activity in cabbage sprouts. Int. J. Food Sci. Technol. 50: 1839-1846.

Zhang, Y. 2004. Cancer-preventive isothiocyanates: Measurement of human exposure and mechanism of action. Mutat. Res. 555: 173-190. 